\title{
Korean red ginseng protects against neuronal damage induced by transient focal ischemia in rats
}

\author{
JU YEON BAN ${ }^{1}$, SUNG WOOK KANG ${ }^{2}$, JONG SEOK LEE ${ }^{3}$, JOO-HO CHUNG ${ }^{2}$, \\ YOUNG GWAN $\mathrm{KO}^{3}$ and HAN SUNG $\mathrm{CHOI}^{3}$ \\ ${ }^{1}$ Department of Pharmacology, College of Dentistry, Dankook University, Cheonan; ${ }^{2}$ Kohwang Medical Research Institute; \\ ${ }^{3}$ Department of Emergency Medicine, School of Medicine, Kyung Hee University, Seoul, Republic of Korea
}

Received November 18, 2011; Accepted December 23, 2011

DOI: $10.3892 /$ etm.2012.449

\begin{abstract}
In the present study, we investigated the neuroprotective effect of Korean red ginseng (KRG) following focal brain ischemia/reperfusion injury, in relation to its antioxidant activities. The middle cerebral artery occlusion/reperfusion (MCAO/R) model in rats was employed. The KRG extract (100 mg/kg, perorally) was administered once daily for 7 days following $\mathrm{MCAO} / \mathrm{R}$. The elevated levels of lipid peroxidation in the $\mathrm{MCAO} / \mathrm{R}$ group were attenuated significantly in the KRG-administered group. The significantly depleted activity of the antioxidant enzymes glutathione peroxidase, superoxide dismutase and catalase was prevented in the KRG-administered group. In the neurobehavioral evaluation expressed as the modified neurological severity score and corner-turn test, the daily intake of KRG showed consistent and significant improvement in the neurological deficits for 7 days following $\mathrm{MCAO} / \mathrm{R}$ injury. These results indicate that KRG has a neuroprotective effect against ischemia/reperfusion brain injury by reducing the level of lipid peroxidation and increasing the endogenous antioxidant enzymatic activity.
\end{abstract}

\section{Introduction}

Stroke is an acute and progressive neurodegenerative disorder. Ischemic stroke is the result of a transient or permanent reduction in cerebral blood flow caused by the occlusion of a cerebral artery via an embolus or local thrombosis (1). The major pathobiological mechanisms of ischemia/reperfusion injury include excitotoxicity, oxidative stress, inflammation, and apoptosis (2,3). These changes are associated with mitochondrial dysfunction and rapid decreases in the ATP level, resulting in free radical generation and lipid peroxidation.

Correspondence to: Professor Han Sung Choi, Department of Emergency Medicine, School of Medicine, Kyung Hee University, Hoegi-dong, Dongdaemun-gu, Seoul 130-701, Republic of Korea E-mail: hsg3748@hanmail.net

Key words: Korean red ginseng, middle cerebral artery occlusion/ reperfusion, antioxidant, lipid peroxidation
Oxidative stress is a crucial factor in cerebral ischemic injury, since the brain consumes a large quantity of oxygen. Reactive oxygen species (ROS) have been indicated as one of the earliest and most significant components of tissue injury following reperfusion of the ischemic organ $(4,5)$. The brain is quite vulnerable to oxidative stress due to its high polyunsaturated fatty acid content, which is particularly susceptible to ROS damage $(6,7)$.

Korean red ginseng (KRG, the steamed root of Panax ginseng C.A. Meyer, Araliaceae) is used frequently as a crude substance that is taken orally as a traditional medicine in Asian countries. It has been reported to show a range of biological activities, such as antitumor, anti-inflammatory, and antistress activities (8-10). These activities have been attributed mainly to saponin, which contains a variety of ginsenosides. There is evidence that the medicinal efficacy of ginseng is closely linked to its protective effects against free radical attack (11). The administration of ginseng to rats has been reported to prevent myocardial ischemia-reperfusion damage induced by hyperbaric oxygen (11). Ginseng has been reported to have protective effects against hepatic oxidative stress caused by exhaustive exercise (12) and against muscle injury and inflammation after eccentric exercise (13). Moreover, it has been reported that ginseng extracts scavenge superoxide radicals, inhibit lipid peroxidation and reduce the level of oxidative DNA damage caused by the Fenton agent $(14,15)$. However, few in vivo studies have examined the neuroprotective effects of KRG on the rat brain affected by ischemia/reperfusion injury. In a previous study, we reported that KRG treatment prevented cerebral ischemic injury induced by middle cerebral artery occlusion/reperfusion (MCAO/R) in rats, which was assessed by staining brain tissue with $2 \%$ 2,3,5-triphenyltetrazolium chloride (16). This study investigated the neuroprotective effect of KRG following a focal brain ischemia/reperfusion injury and focused on the antioxidant activities of KRG.

\section{Materials and methods}

Animals and treatments. Thirty Sprague-Dawley rats weighing $300 \pm 10$ g (mean \pm standard deviation, 9 weeks old) were used. The animals were purchased from Samtako Bio Korea Co., Ltd. (Kyung Gi-Do, Korea). The rats were housed in an environmentally controlled room at $22 \pm 2^{\circ} \mathrm{C}$, with a relative 
humidity of $55 \pm 5 \%$, a 12 -h light/dark cycle, and food and water ad libitum. The study was carried out in accordance with the Korean Academy of Medical Sciences. The animals were placed under general anesthesia and monitored in accordance with the corresponding standard procedures (Laboratory Animal Manual 2000, Korean Academy of Medical Sciences, Seoul, Korea). All experimental procedures were approved by the Kyung Hee University Medical Center Institutional Animal Care and Use Committee, Seoul, Korea.

The animals were divided randomly into three groups: i) the sham operation group $(n=9)$, in which the animal was anesthetized with the left common and external carotid artery exposed but with no arterial ligation or opening; ii) the saline group $(n=12)$, in which the animal underwent the MCAO/R procedure and was treated with normal saline $(6 \mathrm{ml} / \mathrm{kg} / \mathrm{day}$ perorally) for 7 days; and iii) the KRG group ( $\mathrm{K}=9$ ), in which the animal underwent the $\mathrm{MCAO} / \mathrm{R}$ procedure and was treated with KRG (100 mg/kg/day perorally) for 7 days.

Feeding with normal saline or KRG was initiated after the onset of reperfusion injury. The rats were administered with either saline or KRG once daily, perorally, for 7 days. They were treated between 10:00 and 12:00 to avoid any nonspecific effects due to the circadian rhythm.

$K R G$ extract. KRG extract in concentrated form was purchased commercially from the Korea Ginseng Corp. (Daejeon, Korea). It was produced from the roots of fully matured six-year-old Korean ginseng plants. KRG compound contains various ginseng saponin fractions: $30.1 \%$ ginsenoside (G)-Rb1, $13.9 \%$ G-Rb2, 14.4\% G-Rc, 6.1\% G-Rd, $13.9 \%$ G-Re, $4.7 \%$ G-Rf, $11.5 \%$ G-Rg1, 2.6\% G-Rg2, and 2.8\% G-Rg3.

Measurement of regional cerebral blood flow. Each rat was anesthetized with isoflurane (initiated with $5 \%$ and maintained at $2 \%$ ) in a mixture of $30 \%$ oxygen and $70 \%$ nitrous oxide using a vaporizer and face mask. The rat was mounted in the prone position on a dual small animal stereotaxic instrument (David Kopf Instruments, Los Angeles, CA, USA). After a cranial mid-line incision, a 1-mm burr hole was made with a microdrill at both sides, 2-mm-posterior and 5-mm-lateral to the bregma. The rat was then placed in the supine position. A laser Doppler probe was placed on the burr hole under the rat skull, almost contacting the dura mater of the brain surface avoiding the large vessels. The parietal cortical perfusion in the territory of MCA was measured. The laser Doppler probe was connected to a BLF21D laser Doppler flowmeter (Transonic systems Inc., Ithaca, NY, USA), and the values were obtained from $10 \mathrm{~min}$ before the ischemic event until $15 \mathrm{~min}$ after reperfusion. The changes in regional cerebral blood flow (rCBF) are expressed as the percentage of the baseline values. The signals were digitalized and sent to a computer for recording, storage and analysis.

$M C A O / R$. After the laser Doppler probe had been placed on the skull, a left MCAO was induced using the method reported by Longa et al (17) and revised by Lourbopoulos et al (18). The left common carotid artery (CCA) and external carotid artery (ECA) were exposed under an operation microscope followed by electrical coagulation of the ECA branches. The internal carotid artery (ICA) was then dissected to the level of the pterygopalatine artery. After the distal part of the ECA was ligated permanently, silk thread (6-0) was placed loosely around the ECA stump. The CCA and ICA were occluded temporarily using microvascular clips. A small incision was made on the ECA, and $25 \mathrm{~mm}$ nylon thread (4-0) with a rounded tip and a distal cylinder of silicon rubber $(0.30 \mathrm{~mm}$ in diameter $)$ was inserted through the incision. The silk thread around the ECA stump was held tightly to prevent bleeding, and a microvascular clip on the ICA was removed. The nylon thread within ECA was advanced gently through the ICA until laser Doppler flowmetry revealed a sharp decrease in the regional blood flow in the MCA to approximately $20 \%$ of the baseline value, as determined by monitoring. The microvascular clip on the CCA was removed, and the incised skin was closed. Two hours after the induction of ischemia, reperfusion was performed by removing the nylon thread from the ICA under isoflurane anesthesia, as described above. The restoration of blood flow was identified in the operation field primarily, and was confirmed by laser Doppler flowmetry. The animals in the sham operation group were subjected to the same surgical procedure but without insertion of the nylon filament. The rectal temperature was monitored continuously using a thermometer and maintained at $37.0 \pm 0.5^{\circ} \mathrm{C}$ using an electrical blanket and heating lamp throughout the experiment.

Neurological deficits. The neurological deficits were evaluated prior to brain ischemia and at 1,3, and 7 days after MCAO/R injury.

Modified neurological severity scores ( $m N S S)$. The mNSS is a composite of motor, sensory (visual, tactile and proprioceptive), reflex and balance tests. In the severity scores of the injury, the neurological function was graded on a scale of 0-18 (normal score, 0 ; maximal deficit score, 18). One score point was awarded for the inability to perform the test or for the lack of a tested reflex. Thus, a higher score indicates a more severe injury (19).

Corner test. A rat was placed between two boards, each $30 \times 20 \times 1 \mathrm{~cm}$ in size. The edges of the two boards were attached at a $30^{\circ}$ angle with a small opening along the joint between the two boards in order to encourage entry into the corner. The rat was placed between the two angled boards facing the corner and halfway to the corner. When the rat reached the wedge of the corner, both sides of the body were stimulated simultaneously. The animal usually reared and turned either to the right or left. Each animal was tested for 10 trials, and the chosen sides for turning were noted. In the case of ventral turning (i.e., when the animal turned without rearing), the trial was not considered but was performed again at the end of the session. In general, a non-ischemic rat turns either left or right, whereas an ischemic rat preferentially turns toward the non-impaired ipsilateral side. The results were expressed as the percentage of right turns compared to the total number of turns.

Determination of antioxidant enzyme activity and lipid peroxidation. Following the behavioral tests, the animals were sacrificed and their brains were removed quickly and homogenized. The supernatant containing the crude membrane was used to estimate the malondialdehyde (MDA) level. The post-mitochondrial supernatant was used to examine antioxidant enzyme activities and estimate the protein level. The 
protein concentration was determined according to the method described by Lowry et al (20) using purified bovine serum albumin as the standard.

The level of oxidative stress was assessed by estimating the level of MDA, considered an indicator of lipid peroxidation. The MDA concentration was assayed using a chromogenic assay (Bioxytech LPO-586, Oxis International Inc., Portland, OR, USA). This assay measures the free and protein-bound MDA without undue interference from the other lipid peroxidation products. The standard curves for the $0-20 \mu \mathrm{mol} / 1$ range were prepared for each assay using the chromogen supplied in the kits. The assay has a detection limit of $0.1 \mu \mathrm{mol} / 1$ and an interassay variation of $<5 \%$. The results are expressed as nanomoles of MDA per milligram of protein tissue (nmol mg-1 protein).

The glutathione peroxidase (GPx) activity was estimated using the procedure reported in the study by Mohandas et al (21). The reaction mixture consisted of phosphate buffer (0.05 M, pH 7.0), EDTA (1 mM), sodium azide (1 mM), glutathione reductase ( 1 enzyme units $/ \mathrm{ml})$, glutathione (1 mM), NADPH (0.2 mM), $\mathrm{H}_{2} \mathrm{O}_{2}(0.25 \mathrm{mM})$ and $0.1 \mathrm{ml}$ of the post-mitochondrial supernatant in a final volume of $2 \mathrm{ml}$. The optical density was recorded spectrophotometrically at $340 \mathrm{~nm}$. One unit of the enzyme was defined as the number of micromoles of reduced NADPH oxidized per minute. The GPx activity was expressed as units per milligram of protein (units $\mathrm{mg}^{-1}$ protein).

The superoxide dismutase (SOD) activity was measured using the Beauchamp and Fridovich method (22). The total reaction mixture consisting of phosphate buffer $(0.5 \mathrm{M}$, $\mathrm{pH}$ 7.4), post-mitochondrial supernatant, xanthine (1 $\mathrm{mM})$, and NBT $(57 \mu \mathrm{M})$ was incubated for $15 \mathrm{~min}$ at room temperature, and the reaction was initiated by adding xanthine oxidase (50 $\mathrm{mU})$. The reaction rate was measured by recording the change in the absorbance at $550 \mathrm{~nm}$. The SOD activity is presented as units $\mathrm{mg}^{-1}$ protein.

The catalase (CAT) activity was examined using the method reported by Greenwald (23). Briefly, the assay mixture consisted of $0.05 \mathrm{M}$ phosphate buffer ( $\mathrm{pH} 7.0$ ), $0.019 \mathrm{M} \mathrm{H}_{2} \mathrm{O}_{2}$, and post-mitochondrial supernatant in a total volume of $3.0 \mathrm{ml}$. The rate of $\mathrm{H}_{2} \mathrm{O}_{2}$ decomposition was measured spectrophotometrically at $240 \mathrm{~nm}$. The CAT activity was expressed as units $\mathrm{mg}^{-1}$ protein.

Statistical analysis. All data were presented as the means \pm standard error of the mean (SEM). The differences between groups were evaluated by analysis of variance (ANOVA) followed by Student-Newman-Keuls multiple comparison test. Statistical analyses were performed using SPSS for Windows 13.0 software (SPSS Inc., Chicago, IL, USA). The differences between the two groups were examined using a Student's t-test. P-values $<0.05$ were considered to indicate a statistically significant difference.

\section{Results}

Effect of KRG on $r C B F$. Fig. 1 shows the relative changes in $\mathrm{rCBF}$ by laser Doppler flowmetry during and after $2 \mathrm{~h}$ of MCAO. MCAO immediately reduced the $\mathrm{rCBF}$ value to approximately $20 \%$ of the baseline level. The rCBF measure-

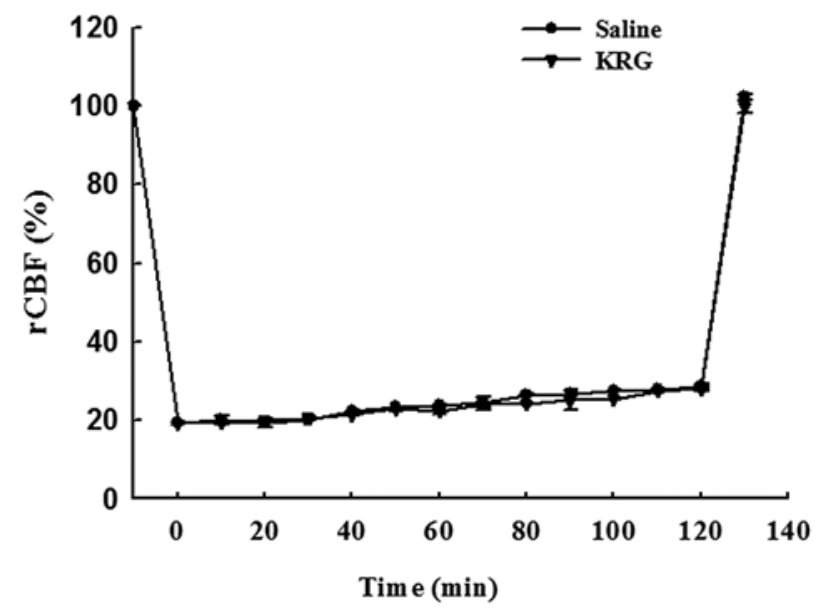

Figure 1. Effect of KRG on regional cerebral blood flow (rCBF). The rCBF decreased similarly in the saline and KRG groups during MCAO, and increased similarly in all the groups following reperfusion $(\mathrm{p}>0.05, \mathrm{n}=8-12)$. The values are reported as the means \pm SEM. KRG, Korean red ginseng; MCAO, middle cerebral artery occlusion.

ments showed similar patterns in all groups. There was no significant difference between the saline and KRG group.

\section{Effect of KRG on the neurological deficits}

$m N S S$. At day 1 after the MCAO/R injury, the rats in the operation groups showed neurological deficits: Saline group (9.8 $\pm 1.0 \mathrm{mNSS}$ points) and KRG group $(10.1 \pm 1.3 \mathrm{mNSS}$ points). There were no significant differences between the operation groups until 3 days after surgery. However, at day 7 after surgery, the mNSS points of the KRG group (5.3 \pm 0.9 points) decreased significantly compared to the saline (8.6 \pm 0.9 points) group ( $\mathrm{p}<0.05)$ (Fig. 2A).

Corner test. The rats in the operation groups showed higher percentages of right turns at one day after MCAO/R injury; saline group $(97.1 \pm 1.1 \%)$ and KRG group $(97.1 \pm 1.3 \%)$. However, there was no significant difference between them. Three days after surgery, the percentage of right turns in the KRG group $(80.0 \pm 3.1 \%)$ was significantly lower than that in the saline group $(89.9 \pm 2.5 \%)(\mathrm{p}<0.05)$. The percentage of right turns in the KRG group $(75.3 \pm 2.7 \%)$ was also significantly lower at 7 days than that in the saline $(87.8 \pm 2.5 \%)$ group $(\mathrm{p}<0.01)$ (Fig. 2B).

Effect of KRG on lipid peroxidation. The effect of KRG on lipid peroxidation was demonstrated by the brain MDA content. The MDA levels were significantly higher in the brain of the saline group $(0.24 \pm 0.12$ vs. $0.38 \pm 0.03, \mathrm{p}<0.01)$. The KRG-treated group followed by $\mathrm{MCAO} / \mathrm{R}$ showed significant decreases in the elevated MDA levels $(0.28 \pm 0.03, \mathrm{p}<0.01)$ (Fig. 3).

Effect of KRG on antioxidant enzymes. Table I lists the changes in the endogenous antioxidant enzymes activities after $\mathrm{MCAO} / \mathrm{R}$ injury. The GPx activity was reduced significantly in the saline groups compared to the sham group (8.71 \pm 1.06 vs. $4.46 \pm 0.69, \mathrm{p}<0.01)$ and recovered to $7.40 \pm 0.76$ in the KRG group $(\mathrm{p}<0.05)$. The CAT and SOD levels were also significantly lower $(\mathrm{p}<0.01)$ in the saline group than those in the sham 
Table I. Effect of KRG on the antioxidant enzymes in the MCAO/R rats.

\begin{tabular}{lccc}
\hline Groups & GPx (units $\mathrm{mg}^{-1}$ protein) & SOD (units $\mathrm{mg}^{-1}$ protein) & CAT (units mg-1 protein) $^{-1.02 \pm 0.34}$ \\
\hline Sham & $8.71 \pm 1.06$ & $2.52 \pm 0.34 .98 \pm 3.07$ \\
Saline & $4.46 \pm 0.69^{\mathrm{a}}$ & $0.79 \pm 0.06^{\mathrm{a}}$ & $24.08 \pm 1.51^{\mathrm{a}}$ \\
KRG & $7.40 \pm 0.76^{\mathrm{b}}$ & $2.34 \pm 0.36^{\mathrm{c}}$ & $32.40 \pm 1.69^{\mathrm{c}}$ \\
\hline
\end{tabular}

Each antioxidant enzyme was measured from the homogenate of whole brain withdrawn at 7 days after ischemia/reperfusion. The values are reported as the means \pm SEM. ${ }^{\mathrm{a}} \mathrm{p}<0.01$ vs. sham group. ${ }^{\mathrm{b}} \mathrm{p}<0.05,{ }^{\mathrm{c}} \mathrm{p}<0.01 \mathrm{vs}$. saline group. MCAO/R, middle cerebral artery occlusion/reperfusion; GPx, glutathione peroxidage; SOD, superoxide dismutase; CAT, catalase; KRG, Korean red ginseng.

A

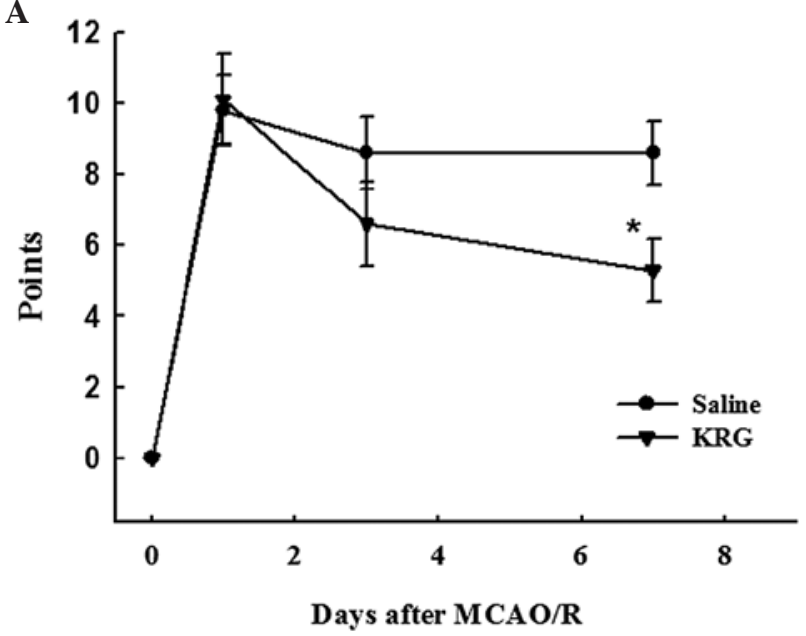

B

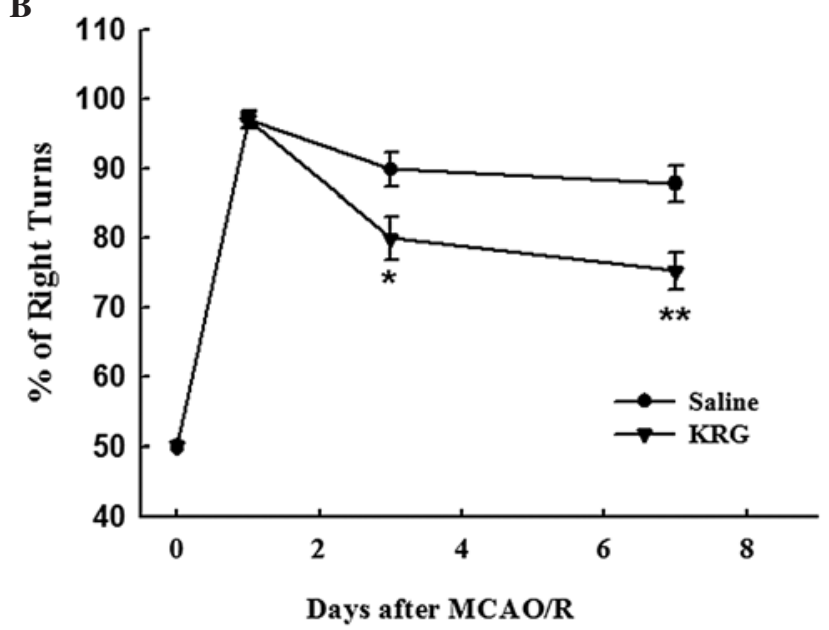

Figure 2. Effect of KRG on neurological deficits. Neurological deficits were evaluated in the saline and KRG group immediately before and 1, 3, and 7 days after MCAO/R using (A) the modified neurological severity scores (mNSS) graded on a scale of 0 to 18 (0, no neurologic deficit; 18, maximal deficit) and (B) the corner test. The values are reported as the means \pm SEM.; $n=8-12$. ${ }^{*} \mathrm{p}<0.05,{ }^{* *} \mathrm{p}<0.01$ vs. saline group. KRG, Korean red ginseng; MCAO/R, middle cerebral artery occlusion/reperfusion.

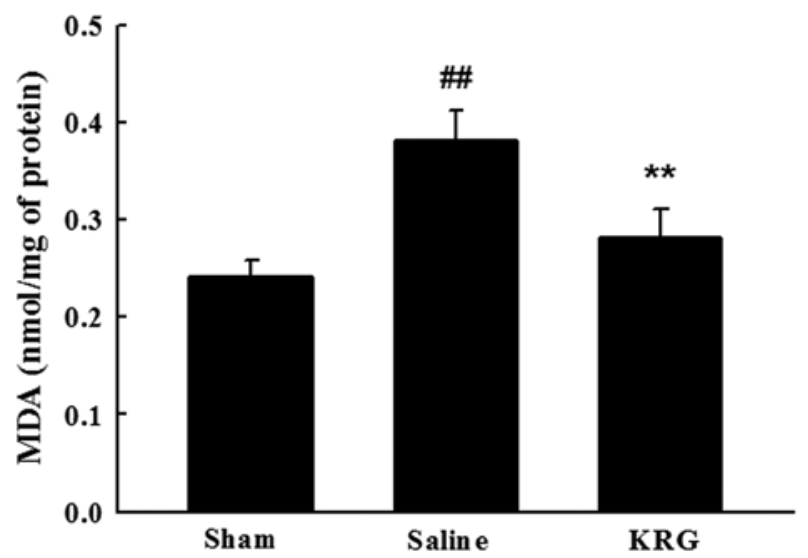

Figure 3. Effect of KRG on lipid peroxidation (MDA). The level of MDA was measured from the supernatant of the whole brain withdrawn at 7 days after ischemia/reperfusion. The values are reported as the means \pm SEM. ${ }^{\# \#} \mathrm{p}<0.01$ vs. sham group; ${ }^{*} \mathrm{p}<0.05,{ }^{* *} \mathrm{p}<0.01$ vs. saline group. KRG, Korean red ginseng; MDA, malondialdehyde.

group, and their activities were restored significantly in the KRG group compared to the saline group $(\mathrm{p}<0.01)$.

\section{Discussion}

Thrombolytic therapy with intravenous recombinant tissue plasminogen activator (rt-PA) has been widely administered for ischemic stroke (must be administered within $3 \mathrm{~h}$ of symptom onset), but its extended implementation is limited (24). It may result in high risk of intracerebral hemorrhage. Therefore, it has been a major challenge to develop effective therapeutics for stroke. In this study, we examined the potential role for clinical applications of KRG in the treatment of ischemic stroke. We used the MCAO model with reperfusion, which mimics many features of stroke in humans (17).

Oxidative stress promotes lipid peroxidation and alters the antioxidant defense system in the brain tissue under ischemic conditions (25). The brain is quite susceptible to oxidative stress due to its environment with non-heme iron, which is involved catalytically in the production of free radicals (26). The close relationship between oxidative stress and cerebral ischemia has generated considerable interest in the development of antioxidant therapies to combat ischemia-induced damage $(27,28)$. Our results showed that the administration of $\mathrm{KRG}$ ameliorated brain injury subsequent to $\mathrm{MCAO} / \mathrm{R}$ in rats. The antioxidant and neuroprotective potential of KRG may 
be responsible for this effect by reducing the level of oxidative stress. These findings are in agreement with those from previous studies $(29,30)$.

Lipids are the macromolecules most susceptible to oxidative stress, which play a major role in the production of MDA (31). The level of MDA in the homogenate of the whole brain of rats was measured to evaluate the oxidative stress induced by $\mathrm{MCAO} / \mathrm{R}$. Our results showed that treatment with KRG extract markedly reduced the MDA level in the brain tissue affected by MCAO/R injury. This indicates that KRG is effective in decreasing the oxidative stress of MCAO/R.

The presence of numerous antioxidant enzymes in the brain, including GPx, SOD, and CAT, protects these tissues from oxidative damage caused by the formation of free radicals (6). GPx plays a key role in removing the excessive free radicals and hydroperoxides, and is a major defense system against oxidative stress in the brain (32). SOD reacts with superoxide radicals to form $\mathrm{H}_{2} \mathrm{O}_{2}$ and $\mathrm{CAT}$, which is found at very low levels in the brain and detoxifies $\mathrm{H}_{2} \mathrm{O}_{2}$ into $\mathrm{H}_{2} \mathrm{O}$. Oxidative stress reduces the activity of these enzymes. Therefore, this study examined the endogenous antioxidant enzymes, such as GPx, SOD, and CAT, which were decreased significantly following ischemia/reperfusion injury. Administration of the KRG extract increased the activity of endogenous antioxidant enzymes to the control levels. The activity of the KRG extract appears to work by restoring the altered antioxidant enzyme activity and decreasing the rate of lipid peroxidation in the brain induced by MCAO/R. Lee et al (33) reported that the long-term administration of ginseng protected the liver cytosolic SOD, GPx, and CAT from age-related deterioration, and antioxidant properties have been reported in ischemia/reperfusion injury in the rat brain (34).

$\mathrm{MCAO} / \mathrm{R}$ causes behavioral deficits in the rat brain, possibly by generating free radicals. The mNSS and corner test were used to measure the neurobehavioral changes. The mNSS is sensitive to unilateral cortical injury as it reflects multiple asymmetries, including postural, sensory and limbuse asymmetries. The corner test was performed to detect the sensorimotor and postural asymmetries. In this study, the rats in the operation groups showed neurological deficits following MCAO/R injury. The impairment of the neurological function reached a maximum at $24 \mathrm{~h}$ after $\mathrm{MCAO} / \mathrm{R}$, and improved progressively at 7 days after $\mathrm{MCAO} / \mathrm{R}$. At day 7 after $\mathrm{MCAO} / \mathrm{R}$, the neurological outcome of the KRG group was notably ameliorated compared to the saline group. These results indicate that $\mathrm{KRG}$, which is a potent antioxidant, prevents the neurobehavioral deficits in the animals by scavenging free radicals. Several authors have also reported that certain antioxidants, including oxyresveratrol and rutin, promote the improvement of neurological deficits by diminishing the level of oxidative stress $(35,36)$. Moreover, it has been reported that glutathione depletion in the rat brain affects neurobehavioral activity (37), indicating a correlation between oxidative stress and behavioral impairment following MCAO injury.

Ginseng has antioxidant activity. Ginseng extract has been reported to inhibit metal-induced lipid peroxidation (38), the progression of renal failure by scavenging radicals, human low-density lipoprotein oxidation and nitric oxide synthase expression in the hippocampus of streptozocininduced diabetic rats (39). The administration of ginseng to rats protected neurons in the rat brain from cerebral ischemia-induced injury (40). Shah et al (41) reported that Korean ginseng tea offered marked protection against ischemia/ reperfusion-induced brain damage, as evidenced by significant reversal of enzymatic alterations. However, these studies focused on the effect of white ginseng and not red ginseng. Of the two types of ginseng, white ginseng is air-dried ginseng, and red ginseng is produced by steaming raw ginseng at $98-100^{\circ} \mathrm{C}$ for $2-3 \mathrm{~h}$. Red ginseng has superior pharmacological activity to white ginseng. The improved biological activities of red ginseng result from changes in the chemical constituents caused by the steaming treatment. Ginseng saponins, referred to as ginsenosides, are believed to play a pharmacologically significant role. Several studies have reported new ginsenosides from red ginsengs that are not normally found in raw ginseng $(42,43)$. The major components of red ginseng are ginsenosides Rg3 and Rb1 (44). Rg3 protects the brain from cerebral ischemia-induced injury by reducing lipid peroxidase, scavenging free radicals and improving the energy metabolism (45). It has been reported that $\mathrm{Rb} 1$ prevents ischemic neuronal death induced by transient cerebral ischemia through a mechanism related to the increased expression of the antiapoptotic genes and modulated expression of the glial cell-derived neurotrophic factor (GDNF) (46). Rh2 and Re also protect the brain from ischemic and reperfusion injuries $(47,48)$. Therefore, the observed prevention of MCAO-induced cerebral ischemic injury by the KRG extract may be due to the beneficial effects of the major active components, such as ginsenosides Rg3 and Rb1.

In conclusion, $\mathrm{KRG}$ protects neurons from MCAO-induced ischemic brain damage, which may be due to the inhibition of neurological deficit and lipid peroxidation, as well as to an increase in the endogenous antioxidant defense enzymes. These results indicate that KRG may be a beneficial intervention in the treatment of ischemic stroke, and highlight the clinical applications of KRG in the treatment of ischemia/reperfusion damage.

\section{References}

1. Dirnagl U, Iadecola C and Moskowitz MA: Pathobiology of ischaemic stroke: an integrated view. Trends Neurosci 22: 391-397, 1999.

2. Shin WH, Park SJ and Kim EJ: Protective effect of anthocyanins in middle cerebral artery occlusion and reperfusion model of cerebral ischemia in rats. Life Sci 79: 130-147, 2006.

3. Mehta SL, Manhas N and Raghubir R: Molecular targets in cerebral ischemia for developing novel therapeutics. Brain Res Rev 54: 34-66, 2007.

4. Kelly PJ, Morrow JD, Ning M, et al: Oxidative stress and matrix metalloproteinase-9 in acute ischemic stroke: the Biomarker Evaluation for Antioxidant Therapies in Stroke (BEAT-Stroke) study. Stroke 39: 100-104, 2008.

5. Wu HW, Li HF, Wu XY, Zhao J and Guo J: Reactive oxygen species mediate ERK activation through different Raf-1dependent signaling pathways following cerebral ischemia. Neurosci Lett 432: 83-87, 2008.

6. Cui K, Luo X, Xu K and Ven Murthy MR: Role of oxidative stress in neurodegeneration: recent developments in assay methods for oxidative stress and nutraceutical antioxidants. Prog Neuropsychopharmacol Biol Psychiatry 28: 771-799, 2004.

7. Halliwell B: Role of free radicals in the neurodegenerative diseases: therapeutic implications for antioxidant treatment. Drugs Aging 18: 685-716, 2001.

8. Wu JY, Gardner BH, Murphy CI, et al: Saponin adjuvant enhancement of antigen-specific immune responses to an experimental HIV-1 vaccine. J Immunol 148: 1519-1525, 1992. 
9. Sato K, Mochizuki M, Saiki I, Yoo YC, Samukawa K and Azuma I: Inhibition of tumor angiogenesis and metastasis by a saponin of Panax ginseng, ginsenoside-Rb2. Biol Pharm Bull 17: 635-639, 1994

10. Kaneko $\mathrm{H}$ and Nakanishi K: Proof of the mysterious efficacy of ginseng: basic and clinical trials: clinical effects of medical ginseng, korean red ginseng: specifically, its anti-stress action for prevention of disease. J Pharmacol Sci 95: 158-162, 2004.

11. Maffei Facino R, Carini M, Aldini G, Berti F and Rossoni G: Panax ginseng administration in the rat prevents myocardial ischemia-reperfusion damage induced by hyperbaric oxygen: evidence for an antioxidant intervention. Planta Med 65: 614-619, 1999.

12. Voces J, Alvarez AI, Vila L, Ferrando A, Cabral de Oliveira C and Prieto JG: Effects of administration of the standardized Panax ginseng extract G115 on hepatic antioxidant function after exhaustive exercise. Comp Biochem Physiol C Pharmacol Toxicol Endocrinol 123: 175-184, 1999.

13. Cabral de Oliveira AC, Perez AC, Merino G, Prieto JG and Alvarez AI: Protective effects of Panax ginseng on muscle injury and inflammation after eccentric exercise. Comp Biochem Physiol C Toxicol Pharmacol 130: 369-377, 2001.

14. Keum YS, Park KK, Lee JM, et al: Antioxidant and anti-tumor promoting activities of the methanol extract of heat-processed ginseng. Cancer Lett 150: 41-48, 2000.

15. Kitts DD, Wijewickreme AN and Hu C: Antioxidant properties of a North American ginseng extract. Mol Cell Biochem 203: $1-10,2000$.

16. Lee JS, Choi HS, Kang SW, et al: Therapeutic effect of Korean red ginseng on inflammatory cy tokines in rats with focal cerebral ischemia/reperfusion injury. Am J Chin Med 39: 83-94, 2011.

17. Longa EZ, Weinstein PR, Carlson S and Cummins R: Reversible middle cerebral artery occlusion without craniectomy in rats. Stroke 20: 84-91, 1989.

18. Lourbopoulos A, Karacostas D, Artemis N, Milonas I and Grigoriadis N: Effectiveness of a new modified intraluminal suture for temporary middle cerebral artery occlusion in rats of various weight. J Neurosci Methods 173: 225-234, 2008.

19. Chen J, Li Y, Wang L, Zhang Z, Lu D, Lu M and Chopp M: Therapeutic benefit of intravenous administration of bone marrow stromal cells after cerebral ischemia in rats. Stroke 32: 1005-1011, 2001

20. Lowry OH, Rosebrough NJ, Farr AL and Randall RJ: Protein measurement with the Folin phenol reagent. J Biol Chem 193 265-275, 1951.

21. Mohandas J, Marshall JJ, Duggin GG, Horvath JS and Tiller DJ: Low activities of glutathione-related enzymes as factors in the genesis of urinary bladder cancer. Cancer Res 44: 5086-5091, 1984

22. Beauchamp C and Fridovich I: Superoxide dismutase: improved assays and an assay applicable to acrylamide gels. Anal Biochem 44: 276-287, 1971.

23. Greenwald RA: CRC Handbook of methods for oxygen radical research. In: Catalase activity. CRC Press, Boca Raton, FL, pp283-284, 1985.

24. Semplicini A, Benetton V, Macchini L, et al: Intravenous thrombolysis in the emergency department for the treatment of acute ischaemic stroke. Emerg Med J 25: 403-406, 2008.

25. Thiyagarajan M and Sharma SS: Neuroprotective effect of curcumin in middle cerebral artery occlusion induced focal cerebral ischemia in rats. Life Sci 74: 969-985, 2004.

26. Yousuf S, Salim S, Ahmad M, Ahmed AS, Ansari MA and Islam F: Protective effect of Khamira Abresham Uood Mastagiwala against free radical induced damage in focal cerebral ischemia. J Ethnopharmacol 99: 179-184, 2005.

27. Simonyi A, Wang Q, Miller RL, Yusof M, Shelat PB, Sun AY and Sun GY: Polyphenols in cerebral ischemia: novel targets for neuroprotection. Mol Neurobiol 31: 135-147, 2005.

28. Ikeda K, Negishi $\mathrm{H}$ and Yamori Y: Antioxidant nutrients and hypoxia/ischemia brain injury in rodents. Toxicology 189: 55-61, 2003 .
29. Kang KS, Kim HY, Pyo JS and Yokozawa T: Increase in the free radical scavenging activity of ginseng by heat-processing. Biol Pharm Bull 29: 750-754, 2006.

30. Bae EA, Hyun YJ, Choo MK, Oh JK, Ryu JH and Kim DH: Protective effect of fermented red ginseng on a transient focal ischemic rats. Arch Pharm Res 27: 1136-1140, 2004.

31. Nazam Ansari M, Bhandari U, Islam F and Tripathi CD: Evaluation of antioxidant and neuroprotective effect of ethanolic extract of Embelia ribes Burm in focal cerebral ischemia/ reperfusion-induced oxidative stress in rats. Fundam Clin Pharmacol 22: 305-314, 2008

32. Imam SZ and Ali SF: Selenium, an antioxidant, attenuates methamphetamine-induced dopaminergic toxicity and peroxynitrite generation. Brain Res 855: 186-191, 2000.

33. Lee H, Kim D and Chang C: Antioxidant effects of Korean red ginseng components on the antioxidant enzymes activity and liver peroxidation in the liver of mouse treated with paraquat. J Ginseng Res 23: 182-189, 1999.

34. Zhang Y and Liu T: Protective effects of total saponins of $P$. ginseng on ischemia-reperfusion injury in rat brains. Chin $J$ Pharmacol Toxicol 8: 7-12, 1994.

35. Khan MM, Ahmad A, Ishrat T, et al: Rutin protects the neural damage induced by transient focal ischemia in rats. Brain Res 1292: 123-135, 2009.

36. Andrabi SA, Spina MG, Lorenz P, Ebmeyer U, Wolf G and Horn TF: Oxyresveratrol (trans-2,3',4,5'-tetrahydroxystilbene) is neuroprotective and inhibits the apoptotic cell death in transient cerebral ischemia. Brain Res 1017: 98-107, 2004

37. Cruz-Aguado R, Almaguer-Melian W, Diaz CM, Lorigados L and Bergado J: Behavioral and biochemical effects of glutathione depletion in the rat brain. Brain Res Bull 55: 327-333, 2001.

38. Zhang D, Yasuda T, Yu Y, Zheng P, Kawabata T, Ma Y and Okada S: Ginseng extract scavenges hydroxyl radical and protects unsaturated fatty acids from decomposition caused by ironmediated lipid peroxidation. Free Radic Biol Med 20: 145-150, 1996.

39. Chang HK, Jang MH, Lim BV, et al: Administration of Ginseng radix decreases nitric oxide synthase expression in the hippocampus of streptozotocin-induced diabetic rats. Am J Chin Med 32: 497-507, 2004.

40. Kim YO, Kim HJ, Kim GS, et al: Panax ginseng protects against global ischemia injury in rat hippocampus. J Med Food 12: 71-76, 2009.

41. Shah ZA, Gilani RA, Sharma P and Vohora SB: Cerebroprotective effect of Korean ginseng tea against global and focal models of ischemia in rats. J Ethnopharmacol 101: 299-307, 2005.

42. Kasai R, Besso H, Tanaka O, Saruwatari Y and Fuwa T: Saponins of red ginseng. Chem Pharm Bull 31: 2120-2125, 1983.

43. Baek NI, Kim DS, Lee YH, Park JD, Lee CB and Kim SI Ginsenoside $\mathrm{Rh} 4$, a genuine dammarane glycoside from Korean red ginseng. Planta Med 62: 86-87, 1996.

44. Kitagawa I, Yoshikawa M, Yoshihara M, Hayashi $\mathrm{T}$ and Taniyama T: Chemical studies of crude drugs (1). Constituents of Ginseng radix rubra. Yakugaku Zasshi 103: 612-622, 1983.

45. Tian J, Fu F, Geng M, et al: Neuroprotective effect of 20(S)-ginsenoside Rg3 on cerebral ischemia in rats. Neurosci Lett 374: 92-97, 2005.

46. Yuan QL, Yang CX, Xu P, et al: Neuroprotective effects of ginsenoside $\mathrm{Rbl}$ on transient cerebral ischemia in rats. Brain Res 1167: 1-12, 2007.

47. Zhou XM, Cao YL and Dou DQ: Protective effect of ginsenosideRe against cerebral ischemia/reperfusion damage in rats. Biol Pharm Bull 29: 2502-2505, 2006

48. Park EK, Choo MK, Oh JK, Ryu JH and Kim DH: Ginsenoside $\mathrm{Rh} 2$ reduces ischemic brain injury in rats. Biol Pharm Bull 27 433-436, 2004 